\title{
ESPECIES NUEVAS DE AVISPAS DE LAS ANTILLAS (HYMENOPTERA: TRIGONALIDAE, CRABRONIDAE, SPHECIDAE)
}

\author{
Julio A. Genaro
}

Investigador asociado del Museo Nacional de Historia Natural "Prof. Eugenio de Jesús Marcano". Calle César Nicolás Penson, Plaza de la Cultura, Santo Domingo.

República Dominicana. polimita@hotmail.com.

\section{RESUMEN}

Se describen los siguientes táxones antillanos nuevos para la ciencia: Taeniogonalos estebani (Trigonalidae, hembra y macho, Hispaniola); Cerceris ayti (macho, Hispaniola), C. surieli (Crabronidae, hembra y macho, Hispaniola); Isodontia poeyi antillana (Sphecidae, hembra, Hispaniola y Puerto Rico) y Pseneo alayoni (hembra, Hispaniola). Isodontia constituye el primer registro del género para la Hispaniola y Puerto Rico, mientras Pseneo para la Hispaniola. Se resume la información conocida para las especies antillanas de Cerceris referente a distribución, presas conocidas y su pertenencia a los grupos creados por Scullen $(1965,1972)$.

Palabras clave: taxonomía, Taeniogonalos, Cerceris, Isodontia, Pseneo, primeros registros, Cuba, Hispaniola, Puerto Rico.

\section{NEW SPECIES OF WASPS FROM THE ANTILLES (HYMENOPTERA: TRIGONALIDAE, CRABRONIDAE, SPHECIDAE)}

\section{ABSTRACT}

The following new Antillean taxa are described: Taeniogonalos estebani (Trigonalidae, female and male, Hispaniola); Cerceris ayti (male, Hispaniola), C. surieli (Crabronidae, female and male, Hispaniola); Isodontia poeyi antillana (Sphecidae, female, Hispaniola and Puerto Rico) and Pseneo alayoni (Crabronidae, female, Hispaniola). Isodontia constituted the first record of the genus from Hispaniola and Puerto Rico, since Pseneo from Hispaniola. A summary about distribution, prey used to provision the nests and how they fit into the groups created by Scullen $(1965,1972)$ for the Antillean species of Cerceris is presented.

Keywords: taxonomy, Taeniogonalos, Cerceris, Isodontia, Pseneo, first records, Cuba, Hispaniola, Puerto Rico.

\section{INTRODUCCIÓN}

Es conocido el valor de los himenópteros como controladores biológicos, depredadores o polinizadores de muchas especies de plantas. Su estudio en las Antillas dista de estar completo, por lo que se debe comenzar por la taxonomía para poder avanzar en el conocimiento de la ecología, la conducta, la genética molecular y, por consiguiente, en la tarea más inmediata y necesaria de todas: la conservación. 


\title{
OBJETIVO
}

-Describir cuatro especies y una subespecie nuevas de avispas de Cuba, Hispaniola y Puerto Rico.

\section{MATERIALES Y MÉTODOS}

La terminología utilizada para la escultura de la superficie del tegumento sigue a Harris (1979). Las abreviaturas son usadas como sigue: cada tergo o esterno metasomal es llamado $\mathrm{T}$ o $\mathrm{S}$, respectivamente, seguido por un número que lo define específicamente, ejemplo: T1 es el primer tergo y S1 el primer esterno. La densidad de las puntuaciones está dada en términos de la relación entre el diámetro de la puntuación (d) y el inter-espacio (i) entre ellas, como ejemplo $\mathrm{i}=2 \mathrm{~d}$, para demostrar su separación. La longitud de los pelos está en relación con el diámetro del ocelo medio (DOM), por ejemplo $<$ DOM significa que la longitud del pelo es menor que el DOM.

Instituciones donde está depositado el material estudiado o a las cuales se hace referencia en el texto: CNC, Canadian National Collection of Insects, Arachnids and Nematodes, Ottawa; JAG, colección personal del autor; FSCA, Florida State Collection of Arthropods Gainesville, Florida, Estados Unidos; MCZ, The Louis Agassiz Museum of Comparative Zoology, Harvard, Estados Unidos; MNHNCu, Museo Nacional de Historia Natural de Cuba; MNHNSD, Museo Nacional de Historia Natural "Prof. Eugenio de Jesús Marcano", República Dominicana; NMNH, National Museum of Natural History, Estados Unidos, Institución Smithsoniana.

\author{
TAXONOMÍA \\ Familia Trigonalidae \\ Subfamilia Trigonalinae \\ Taeniogonalos estebani sp. nov.
}

(figs. 1-5; 7-10)

Diagnosis. Tegumento negro con marcas blancas; T. gundlachii (Cresson, 1865) tiene las marcas corporales amarillas y están más extendidas; por ejemplo, el escutelo es completamente amarillo, mientras en la especie nueva no presenta marcas. La armadura, solo presente en la hembra, está poco diferenciada, observándose una pequeña muesca y un dientecito a cada lado en la zona central del margen posterior de S2 (fig. 5), mientras en T. gundlachii es abultada y se eleva en la porción media de S2 (fig.6).

Diagnosis. Tegument black with white marks; the light colored markings are yellow and being more extensive in $T$. gundlachii (Cresson, 1865), ie. the new species have the scutellum all black. The armature, only found in the female, is not so differentiated into a separate bifid tooth, but rather the whole hind edge of S2 is evenly curved (fig. 5), i.e., in T. gundlachii the armature rises up from the medial portion of the apex of S2 (fig. 6). 


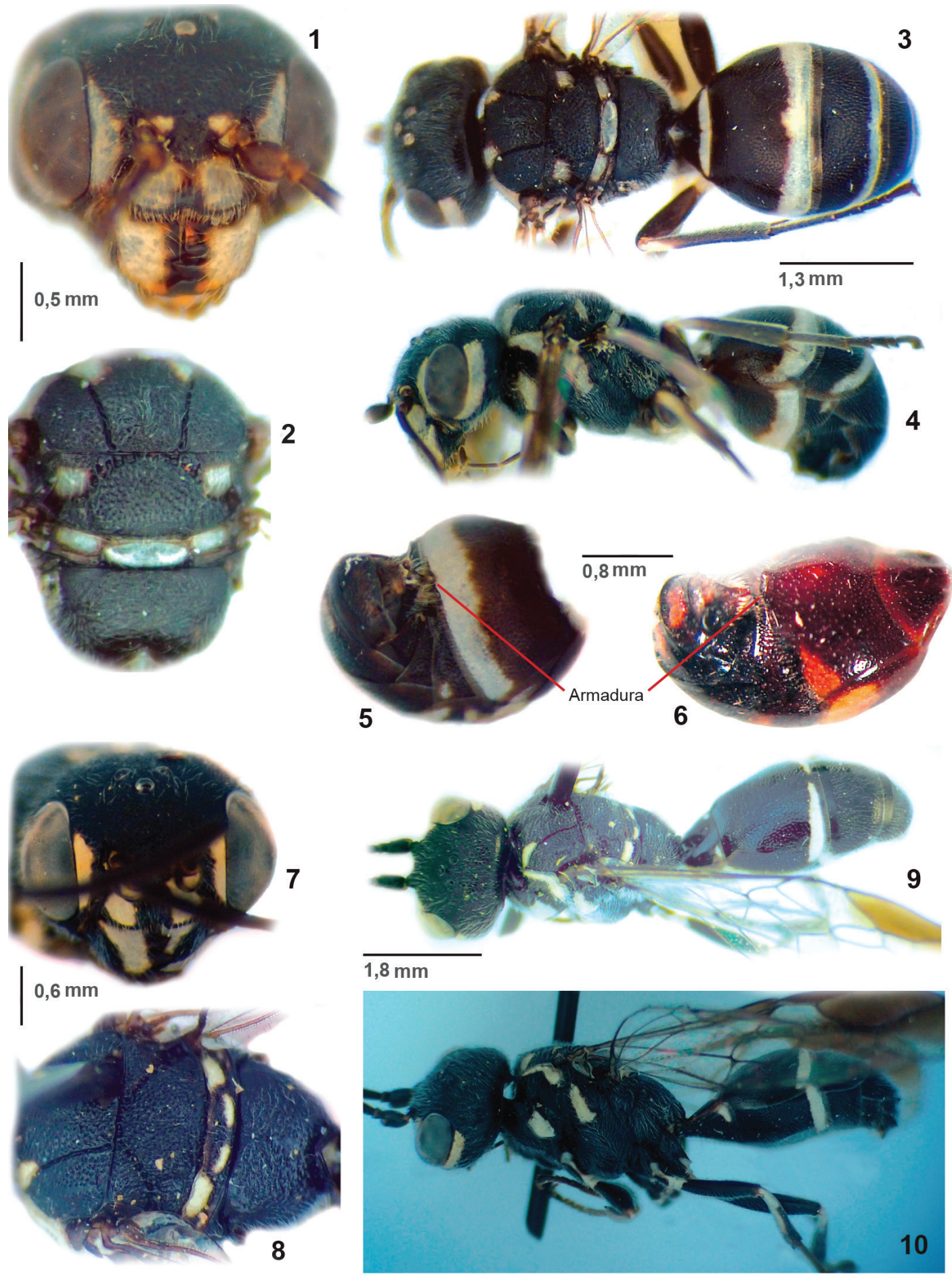

FIGURAS 1-10. Taeniogonalos spp. 1-5. T. estebani sp. nov., hembra: 1, vista frontal de la cabeza. 2, vista dorsal del mesosoma. 3, hábito dorsal. 4, hábito lateral. 5, vista ventral del metasoma, mostrando la armadura. 6. T. gundlachii hembra, vista ventral del metasoma, mostrando la armadura. 7-10. T. estebani sp. nov., macho: 7, vista frontal de la cabeza. 8 , vista dorsal del mesosoma. 9, hábito semi-dorsal. 10, hábito lateral. 


\section{DESCRIPCIÓN}

\section{HEMBRA}

Longitud corporal total aproximada: $5.2 \mathrm{~mm}$. Longitud ala anterior: $4.2 \mathrm{~mm}$.

Coloración. Tegumento negro excepto en las siguientes áreas, que son blancas. Cabeza: una mancha en elevación supra-antenal; banda en área paraocular; superficie dorsal de las mandíbulas (excepto los dientes); ocupando casi todo el clípeo (interrumpida en el centro; fig. 1); gena, a lo largo del ojo. Mesosoma: pronoto; dos manchas triangulares que se extienden desde el margen anterior; mesoescuto; axilas; metanoto; parte del propleuron; mancha en extremo dorsal del mesepisterno (fig. 2). Alas: venación castaño oscuro; mitad anterior ahumada, color castaño. Patas: coxas y trocánteres; área ventral tibias, reduciendo su extensión hacia la tibia posterior, donde solo se observa en el extremo basal. Metasoma: banda apical T1-T3 (ligeramente más ancha en T2); bandas en T4 y T5 reducen su longitud y no llegan a los lados; banda apical en S1; una mancha apical a cada lado de S2 (figs. 3 y 4).

Esculturación. Cabeza: mandíbulas con puntuaciones alargadas, irregulares; clípeo irregularmente puntuado $(\mathrm{i}=0.5-3 \mathrm{~d})$, puntuaciones grandes y pequeñas, área central impuntuada; frente, vértex y gena puntuado-reticulado. Mesosoma: mesoescutelo, escutelo puntuado-reticulado, con puntuaciones mayores en escutelo; metanoto con algunas puntuaciones pequeñas, aisladas; propodeo con surcos semi-paralelos longitudinales, que en ocasiones se ramifican y llegan hasta la zona media, donde aparecen puntuaciones grandes irregulares; zona central del área basal del propodeo brillante, ligeramente arrugada con puntuaciones aisladas. Propleuron con puntuaciones variables $(\mathrm{i}=0.5-3 \mathrm{~d})$; mesepisterno puntuado-reticulado; mesopleuron puntuado-reticulado con surcos transversales semi-paralelos. Metasoma: T1 pulido, liso con algunas micro-puntuaciones; T2-T3 con puntuaciones variables $(\mathrm{i}=0-3 \mathrm{~d})$; T4-T6 puntuado-reticulado. Esternos con puntuaciones variables $(i=0-3 d)$.

Pubescencia. Blanca variable $\leq \mathrm{DOM}$, ligeramente esparcida; más larga en mandíbulas y lados del propodeo.

Estructura. Cabeza: número de segmentos antenales, 24; clípeo con muesca central; elevación supra-antenal reducida, formando una protuberancia triangular. Armadura en S2, poco diferenciada, observándose una pequeña muesca en el centro del margen posterior y dos dientecitos poco desarrollados.

\section{MACHO}

Longitud corporal total aproximada: $7.3 \mathrm{~mm}$. Longitud ala anterior: $6.4 \mathrm{~mm}$.

Coloración. Con manchas blancas menos extendidas (figs. 7-10). Igual a la hembra, excepto: ausencia de manchas blancas en elevación supra-antenal, coxas y trocánteres (con negro que cubre casi toda la superficie), T3 y T4; reducida en metanoto; T1 con dos manchas apicales laterales; T2 con banda interrumpida en el medio; S1 con banda apical reducida a una mancha a cada lado; S2 interrumpida en el centro. Alas ahumadas en las siguientes celdillas: costal, parte de la primera radial 1 y tercer sector radial, y primera radial 2.

Esculturación. Puntuaciones más profundas y groseras. Cabeza: puntuaciones menos profundas en áreas de color blanco. Mesosoma: puntuaciones de mesoescuto y escutelo de igual tamaño; propodeo puntuado-reticulado (fig. 8). Metasoma: T2-T3 con línea media impuntuada que se eleva ligeramente a partir de T4 formando una carena. 
Estructura. Muesca del clípeo más pronunciada (fig. 7). Presencia de tiloides, lineales, estrechos en los segmentos antenales 8-16.

Tipos. Holotipo hembra: HISPANIOLA, Dominican Republic, Prov.[incia] Pedernales, 26 km N Cabo Rojo, vii. 1998, 730 m FIT, col. L. Masner, mesic deciduos forest. Taeniogonalos sp. undescribed, det. Carmean, 1993 (CNC); Paratipo macho: República Dominicana, Parque Nacional A. Bermúdez, Antón Sape Bueno, Trillo de la Hispaniola, xii.2008, col. J. A. Genaro (CNC).

Etimología. Dedicada al amigo y eminente taxónomo, especialista en cucarachas Esteban Gutiérrez (MNHNCu).

Distribución. La Hispaniola, Antillas Mayores.

Comentario. Esta constituye la segunda especie de la familia conocida para las Antillas. Previamente se conocía a T. gundlachii de Cuba y Este de Norteamérica (Alayo, 1974; Carmean y Kimsey, 1998). Smith et al. (2012) enmiendan el error del registro anterior para Costa Rica (Carmean y Kimsey, 1998).

D. Carmean (com. pers., 2016; http://www.sfu.ca/ carmean/trig/trigdbase.html) no incluyó la hembra de la Hispaniola en su estudio (Carmean y Kimsey, 1998) debido a la carencia de ejemplares, incluyendo el sexo opuesto y pensó que las marcas claras del tegumento fueron debidas a la preservación en el fluido de la trampa de intercepción.

Familia Crabronidae

Subfamilia Philantinae

Cerceris ayti sp. nov.

(figs.11-13)

Diagnosis. Tegumento negro con marcas amarillas. Propodeo y mesepisterno sin marcas amarillas. Clípeo ligeramente convexo con dos dientecitos en el margen. Una combinación de caracteres que incluyen el patrón de marcas corporales, la estructura del clípeo y la esculturación la separan de otras especies antillanas.

Diagnosis. Black with yellow markings. Propodeum and mesepisternum black without marks. Clypeus slightly extending outward with two denticles on margin. A combination of characters such as pattern of yellow markings, form of the clypeus and sculpturation separates the new taxon from other Antillean species.

\section{DESCRIPCIÓN}

\section{MACHO}

Longitud corporal total aproximada: $12.5 \mathrm{~mm}$. Longitud ala anterior: $11.0 \mathrm{~mm}$.

Coloración. Tegumento negro excepto en las siguientes áreas, que son amarillas (fig. 12): escapo antenal, ventralmente; áreas supraclipeal y paraocular; clípeo; base mandíbulas (fig. 11); pequeña mancha en gena, superiormente; banda en pronoto interrumpida ampliamente en el centro; gran parte de tégula; metanoto; tarsos, tibias, casi todo el fémur posterior, excepto al área apical, la mayor parte de los fémures anterior y medio, excepto el extremo basal, extendiéndose más en el área ventral; mancha en coxa posterior; T1 con dos manchas a cada lado; el resto de los tergos con banda apical, más estrecha en el centro; S2-S3 con manchas pequeñas a cada lado. Alas ligeramente oscurecidas con venación color castaño. 
Esculturación. Clípeo con puntuación irregular, más escasa en el disco; frente, área paraocular y gena puntuado-reticulado. Mesoescutelo con puntuación irregular (i=1-3d), más unida en el disco; escutelo con puntuación irregular $(\mathrm{i}=0.5-3 \mathrm{~d})$, pequeñas y grandes; metanoto con puntuación de menor tamaño; área basal del propodeo con surcos paralelos; superficie posterior del propodeo con esculturación irregular, puntuación alargada incluida en surcos hacia el área basal, quedando puntuación irregular alargada en el resto, combinada con reticulación. Mesepisterno puntuado-reticulado. Tergos con puntuación irregular $(\mathrm{i}=0.5-3 \mathrm{~d})$; esternos con puntuación irregular $(\mathrm{i}=0-3 \mathrm{~d})$. Área pigidial con puntuación robusta, irregular $(\mathrm{i}=0.5-5 \mathrm{~d}$; fig. 13).

Pubescencia. Amarillo claro. Lóbulos de pelos en clípeo ampliamente separados, longitud = DOM y 1.5 DOM (fig. 11). Más larga en gena, propodeo, mesepisterno y T1 (=3 DOM); el resto del cuerpo variable, entre 1-2 DOM.

Estructura. Clípeo ligeramente convexo con dos dientecitos en el margen. Área pigidial más larga que ancha con borde apical ligeramente convexo (fig. 13).

\section{HEMBRA: DESCONOCIDA}

Tipos. Holotipo macho: HISPANIOLA, Port au Prince, Haiti, col. Mann, near stigmosalis (MCZ).

Etimología. Ayti es un término aborigen, que significa tierra áspera, con altas montañas, utilizada para nombrar la isla de La Hispaniola, lugar donde habita la especie nueva. Este nombre fue utilizado en algunos mapas franceses antiguos.

Distribución. La Hispaniola, las Antillas.

Cerceris surieli sp. nov.

(figs. 14-21)

Diagnosis. Tamaño grande, tegumento negro o castaño-rojizo con marcas amarillas. Hembra con margen libre del clípeo con cinco dientecitos en área central; proceso clipeal extendido, con margen anterior emarginado que forma una "v" invertida (figs. 17 y 18); tubérculos mesoesternales presentes (fig. 16). Macho con lóbulo medio del clípeo ligeramente extendido, con tres dientecitos sobre el margen. Cerceris surieli sp. nov. está morfológicamente cercana a $C$. flavocostalis Cresson, 1865 de Cuba, pero una combinación de caracteres como el tamaño corporal, patrón de color, forma del proceso clipeal y placa pigidial la separan de otras especies antillanas.

Diagnosis. Large size, tegument black or ferruginous with yellow markings. Clypeal margin of female with five denticles at the middle; clypeal process extended, with a distinct process with emarginated anterior margin forming an inverted "v" (figs. 17 and 18), and mesosternal tubercles present (fig. 16). Male with medial clypeal lobe slightly extended with three indistinct denticles on margin. Cerceris surieli sp. nov. is related to C. flavocostalis Cresson, 1865 from Cuba. A combination of characters such as corporal size, form of the clypeal process and pigidial plate separates $C$. surieli sp. nov. from other antillean species. 

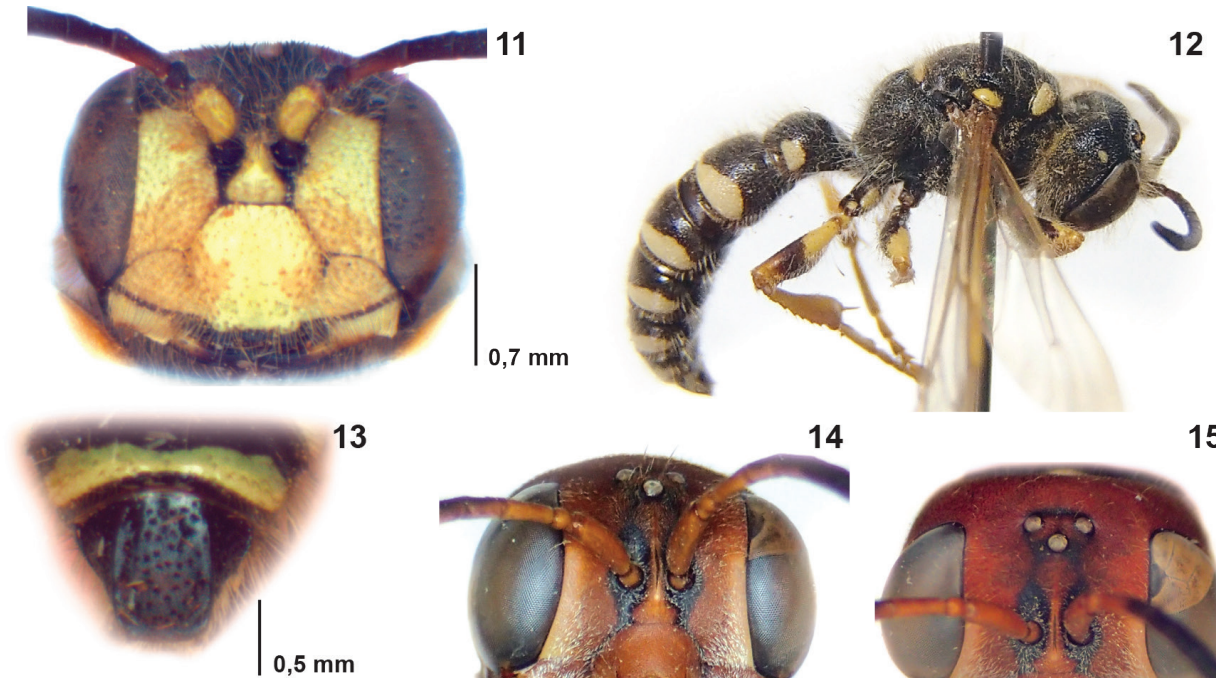

13
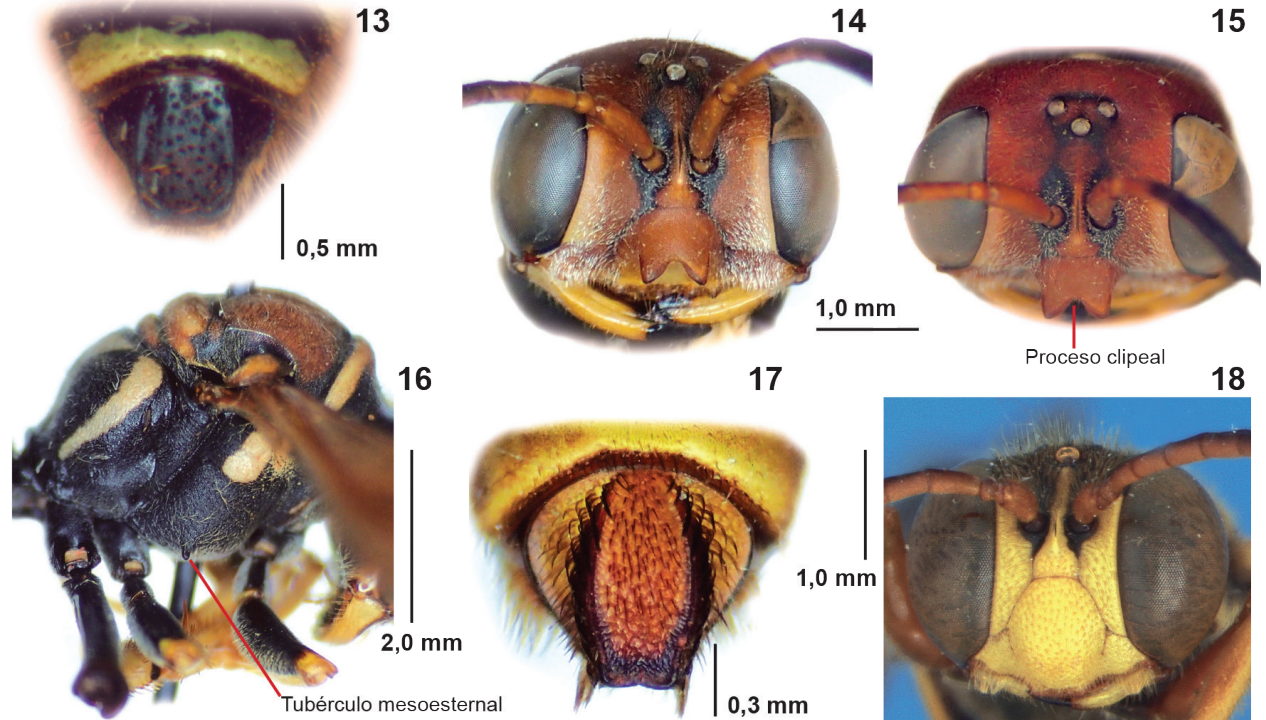

17

18

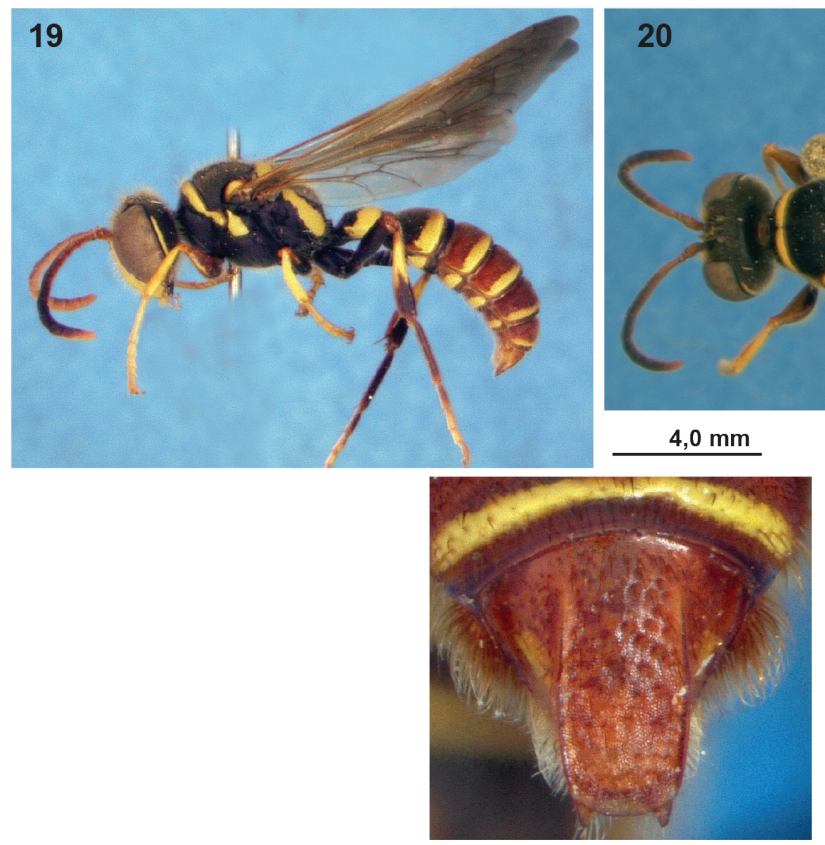

21

$0,6 \mathrm{~mm}$

FIGURAS 11-21. Especies nuevas de Cerceris. 11-13. C. ayti macho: 11, vista frontal de la cabeza. 12, hábito lateral. 13, área pigidial. 14-17. C. surieli hembra: 14, vista frontal de la cabeza. 15, vista dorsal de la cabeza. 16, mesosoma en vista lateral mostrando el tubérculo mesoesternal. 17, área pigidial. 18-21. C. surieli macho: 18, vista frontal de la cabeza. 19, hábito lateral. 20, hábito dorsal. 21, área pigidial. 


\section{DESCRIPCIÓN}

\section{HEMBRA}

Longitud corporal total aproximada: $15.0 \mathrm{~mm}$. Longitud ala anterior: $12.0 \mathrm{~mm}$.

Coloración. Tegumento negro con áreas amarillas y castaño-rojizo. Cabeza (figs. 14 y 15): castaño-rojizo en casi todo el clípeo, frente, proceso clipeal, área supraclipeal, vértex, área central de la gena, tres primeros segmentos antenales. Amarillo claro en mandíbula, excepto ápice y línea sobre margen interno, negros; línea en borde ventral del clípeo; línea a lo largo del margen interno del ojo, en área genal; franja más ancha en área paraocular; área posterior de la gena; tres últimos segmentos antenales. Mesosoma: negro con marcas amarillas en las siguientes áreas: franja estrecha en margen anterior y posterior del propodeo; mancha alargada, muy pequeña en área ventro-lateral del propodeo; franja central en escutelo; casi todo el metanoto; triángulo dorsal del propodeo (excepto margen anterior); una banda longitudinal semicircular, a cada lado, en área basal del propodeo; una mancha en área hipoepimeral; T6 con áreas laterales no ocupadas por la placa pigidial. Áreas castaño-rojizo en franja interrumpida en el centro en margen posterior del pronoto; disco del mesoescuto; mancha central en tégula. Patas negras con amarillo en tarsos; tibias, excepto ápice de la tibia posterior. Alas ahumadas, castaño-amarillento con venas castaño, volviéndose castaño amarillento en margen anterior. Metasoma negro excepto castaño-rojizo en T5 y placa pigidial; banda amarilla en margen posterior de T1-T4, estrechándose en el centro; T3 y T4 con mancha lateral castaño rojizo alargada, interrumpiendo la banda amarilla; en T5 está poco definida. Color de la banda amarilla menos definida en los esternos, ausente en S1, presente en depresión esternal de S3-S5.

Esculturación. Cabeza: clípeo, áreas supraclipeal y paraocular, proceso clipeal, gena, vértex muy finamente punteado-reticulado; puntuaciones aisladas, variables $(\mathrm{i}=0.5-2 \mathrm{~d})$ en margen apical de proceso clipeal y gena. Mesosoma: pronoto, mesoescudo, escutelo y metanoto granulosos, con puntuaciones poco profundas, aisladas, variables; mesepisterno y área basal del propodeo con puntuaciones alargadas, dispuestas en hileras; triángulo dorsal del propodeo impuntuado. Metasoma: tergos y esternos granulosos, con puntuaciones variables, aisladas; depresión esternal-apical en S2-S4 con puntuaciones grandes y pequeñas, igualmente variables. Área pigidial con puntuaciones grandes, arrugándose hacia margen apical.

Pubescencia. Amarillo-amarillo claro variable, excepto castaño sobre T6, rodeando el margen del área pigidial (1.5 DOM). Corta y recostada sobre clípeo, área supraclipeal y frente (>DOM); pelos más largos en propodeo y mesepisterno (2.5-3 MOD). Pelos oscuros cortos saliendo de cada puntuación en área pigidial.

Estructura. Cabeza más ancha que mesosoma. Antena con 12 segmentos; carena interantenal elevada, margen del clípeo con cinco dientecitos en área central; proceso clipeal extendido con margen anterior emarginado formando una "v" invertida (figs. 14 y 15). Tubérculos mesoesternales presentes (fig. 16); ápice del metafémur dilatado. Seis segmentos abdominales; área pigidial con márgenes laterales ligeramente cóncavos, margen apical con muesca central (fig. 17).

\section{MACHO}

Longitud corporal total aproximada: 10.4-13.0 mm. Longitud ala anterior: 9.8-12.0 mm.

Coloración. Tegumento negro o castaño-rojizo en algunas áreas, con manchas amarillas (figs. 19 y 20). El amarillo es más acentuado que en la hembra. Parecido a la hembra, excepto 
por el color amarillo presente en las siguientes áreas. Cabeza (fig. 18): clípeo, áreas paraocular y supraclipeal donde se continúa en una línea que toca el ocelo medio; color menos definido, en área ventral de la antena. Mesosoma: banda variable en metanoto, no se observa en algunos individuos; dos líneas longitudinales, cortas, debajo del triángulo dorsal del propodeo, solo presentes en un ejemplar; dos manchas en mesopleuron, encima de las coxas medias, variables; mancha en pro-coxa (ausente en un ejemplar). Alas menos ahumadas y más amarillentas. Patas negras excepto amarillo en los tarsos, tibias medias y posteriores; casi toda la tibia posterior excepto ápice; ápice de fémures anterior y medio. El fémur anterior es castaño-rojizo, excepto un ejemplar que lo tiene negro; fémur medio tiene áreas donde el negro se sustituye por castañorojizo. Metasoma: T1 negro; T2 negro, excepto castaño-rojizo en un individuo; el resto de los tergos castaño-rojizo, oscureciéndose en los últimos en algunos ejemplares; las bandas apicales amarillas más estrechas que en las hembras; placa pigidial variable, desde castaño-rojizo con algo de amarillo hasta castaño-rojizo oscuro. S1 y S2 negros, el resto de los esternos castañorojizo, aunque en un ejemplar S3 es negro; la banda apical amarilla se estrecha en el centro; mancha amarilla en el disco de S1.

El ejemplar de Barahona está extensivamente manchado de amarillo, presentando marcas ausentes en los otros, lo que demuestra la variabilidad en la presencia y extensión de las manchas en este género.

Esculturación. Puntuaciones mayores y más profundas que en la hembra. Cabeza: clípeo, área supraclipeal y paraocular con puntuaciones variables $(\mathrm{i}=0.5-3 \mathrm{~d})$; gena costulada. Mesosoma: mesoescuto puntuado-reticulado, escutelo con puntuaciones variables $(i=0-1 d)$; metanoto pulido, con puntuaciones grandes y pequeñas aisladas; triángulo dorsal del propodeo con puntuaciones variables ( $\mathrm{i}=0$ - $1 \mathrm{~d}$ ), algo más pequeñas que las del escutelo; área basal del propodeo y mesepisterno con puntuaciones variables en tamaño, más groseras. Metasoma: tergos con puntuaciones grandes variables, más densas en T1 y T2; área pigidial con puntuaciones grandes variables y algunas pequeñas; esternos pulidos, con puntuaciones variables, grandes y pequeñas.

Pubescencia. Como en la hembra, excepto mechón de pelos a ambos lados del clípeo (1 MOD), ocupando un área estrecha del margen del clípeo; los pelos que rodean el área pigidial son claros y menos robustos.

Estructura. Antena con 13 segmentos. Área central del margen del clípeo ligeramente extendida, desde el lóbulo central, con tres dientecitos separados (fig. 18). Tubérculos mesoesternales ausentes; ápice del metafémur menos dilatado que en la hembra. Siete segmentos abdominales. Placa pigidial más larga que ancha, rectangular, con lados ligeramente convexos (fig. 21).

Tipos. Holotipo hembra: HISPANIOLA, República Dominicana, Las Abejas, Sierra de Bahoruco, xi.2005, col. J. A. Genaro (FSCA). Paratipos: Dominican Republic, Prov. La Altagracia, Cueva de Berna, Boca de Yuma, 6.vi.86, colls. R. Miller \& L. Stange (8 machos, FSCA, JAG); Barahona nr. Filipinas, Larimar mine, 1.viii.91, coll. R. E. Woodruff (macho, FSCA).

Etimología. Dedicada al amigo y colega Carlos Suriel (MNHNSD) por sus contribuciones al desarrollo de la zoología antillana, como curador, profesor y editor.

Distribución. La Hispaniola, Antillas Mayores.

Comentario. La hembra tiene coloración compleja, que combina degradaciones de amarillo y castaño-rojizo sobre un fondo negro que también puede cambiar la intensidad. Los sexos están asociados por las similaridades morfológicas. Estos, no fueron recolectados en la misma 
localidad ni juntos en un área de nidificación. Cerceris surieli sp. nov. taxonómicamente está cercana a $C$. flavocostalis y ambas especies se asemejan a avispas del género Polistes (Vespidae Alayo, 1968; obs. pers. del autor).

La Tabla I presenta una lista actualizada de las especies antillanas de Cerceris y algunos elementos de distribución y pertenencia a los grupos taxonómicos creados por Scullen (1965, 1972). Estos grupos incluyen a las especies Norte y Centroamericanas pero no siempre se cumple para la especies antillanas, por lo que deben estudiarse con mayor profundidad para determinar los grupos naturales a que estas pertenecen.

\section{TABLA I.}

Especies antillanas de Cerceris, distribución, presas conocidas y pertenencia a los grupos creados por Scullen $(1965,1972)$.

\begin{tabular}{|c|c|c|c|}
\hline Especies de Cerceris & Distribución & $\begin{array}{c}\text { Grupos especies de } \\
\text { Scullen }(1965,1972)\end{array}$ & $\begin{array}{c}\text { Familia de } \\
\text { presas conocidas }\end{array}$ \\
\hline watlingensis Elliot \& Salbert, 1979 & Las Bahamas & ------- & Curculionidae $^{1}$ \\
\hline cerverae Giner-Marí, 1941 & Cuba & II & Buprestidae $e^{2,3,8}$ \\
\hline festiva Cresson, 1865 & Cuba, Isla de la Juventud & ------- & $\begin{array}{c}\text { Curculionidae,Bruchidae, } \\
\text { Anthribidae }^{2}\end{array}$ \\
\hline flavocostalis Cresson, 1865 & Cuba, Isla de la Juventud & ------- & $?$ \\
\hline hatuey Alayo, 1968 & Cuba & ------- & $?$ \\
\hline trinitaria Alayo, 1968 & Cuba & III & $?$ \\
\hline triangulata Cresson, 1865 & Cuba, Isla de la Juventud & III & $?$ \\
\hline zonata Cresson, 1865 & $\begin{array}{c}\text { Cuba, Isla de la Juventud, } \\
\text { Las Bahamas, Jamaica }\end{array}$ & I & Chrysomelidae ${ }^{2,4}$ \\
\hline farri Scullen, 1970 & Jamaica & $\mathrm{I}$ & $?$ \\
\hline ayti sp. nov & Hispaniola & ------ & $?$ \\
\hline howardevansi Genaro, 2004 & Hispaniola & I & $?$ \\
\hline jeiti Genaro, 2009 & Hispaniola & II & $\begin{array}{l}\text { Chrysomelidae, } \\
\text { Buprestidae }^{5}\end{array}$ \\
\hline laevigata F. Smith, 1856 & Hispaniola & ------ & Curculionidae $^{6}$ \\
\hline surieli sp. nov & Hispaniola & ------ & $?$ \\
\hline krugi Dewitz, 1881 & Puerto Rico, Isla Guana ${ }^{7}$ & ------ & $\begin{array}{c}\text { Attelabidae }^{5} \\
\text { (Curculionoidea) }\end{array}$ \\
\hline victori Genaro, 2009 & Puerto Rico & II & $\begin{array}{l}\text { Chrysomelidae, } \\
\text { Buprestidae }^{5}\end{array}$ \\
\hline nigra Ashmead, 1900 & $\begin{array}{l}\text { St Vicente, Guyana, } \\
\text { Panamá }\end{array}$ & ------- & $?$ \\
\hline
\end{tabular}

Leyenda. 1, Salbert y Elliott (1979); 2, Genaro y Sánchez (1993); 3, Yong y Breto (2015); 4, Elliot et al (1981); 5, Genaro (2009); 6, especie no identificada, primer registro de presa, 7, Snelling (2005) registra con dudas a la especie para la Isla de Guana, como C. margaretella Rohwer, 1915; un ejemplar macho estudiado confirma su presencia para la isla (British Virgin Isl., Guana, 0-80 m, 13-16.vii.1986, cols. S. E. Miller y M. G. Pogue [FSCA ] ); 8, Nelson y Bellamy (2004) en la revisión del género Paratyndaris presentan el registro de una presa en base a material enviado por este autor, re-identificada como $P$. suturalis Fall. --------- carente de información para ser asignado a un grupo o no cumple con las características del grupo. 


\section{Familia Sphecidae \\ Subfamilia Sphecinae \\ Isodontia poeyi antillana ssp. nov.}

(figs. 22-23)

Diagnosis. Tegumento azul metálico. Pubescencia blanca, excepto en clípeo y algunos pelos aislados en cabeza y esternos, negros; frente con algunos pelos bicolores (negro y blanco). En la subespecie nominal la pubescencia es completamente blanca. Clípeo engrosado en el borde libre con pequeña muesca en el área central.

Diagnosis. Metallic blue with white pubescence except clypeus and some scattered hairs on esterna, black (in the nominal subspecies all pubescence is white); frons with some bicolor hairs (black and white); clypeal free margin thickened with a small median notch.
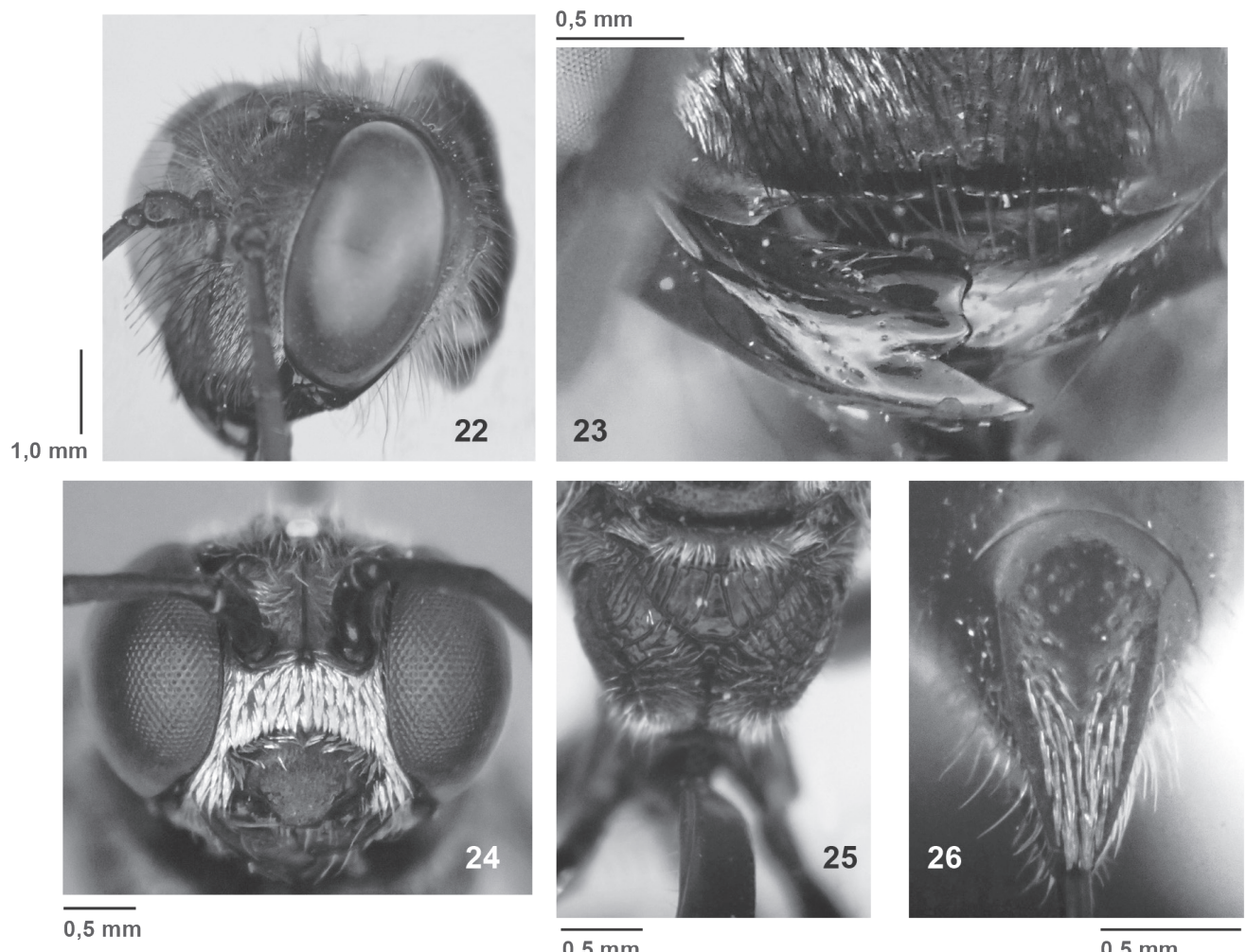

FIGURAS 22-26. Taxones nuevos de avispas. 22-23. Isodontia poeyi antillana hembra: 22, vista semi-frontal de la cabeza. 23, detalle del clípeo y mandíbula. 24-26. Pseneo alayoni: 24, vista frontal de la cabeza. 25, propodeo y peciolo. 26, área pigidial.

\section{DESCRIPCIÓN}

\section{HEMBRA}

Longitud corporal total aproximada: 18.2-21.2 mm. Longitud ala anterior: 16.0-17.5 mm.

Coloración. Tegumento azul metálico; alas castaño rojizo, más oscurecidas apicalmente, con venas castaño oscuro. 
Esculturación. Cabeza: clípeo con puntuaciones variables, grandes, aisladas (i=0-3d); área supraclipeal impuntuada; frente con puntuaciones variables, grandes y pequeñas mezcladas $(\mathrm{i}=0-2.5 \mathrm{~d})$; vértex, gena con puntuaciones variables grandes y pequeñas $(\mathrm{i}=0-3 \mathrm{~d})$, menos frecuentes cerca del margen del ojo. Mesosoma: pronoto con puntuaciones variables; mesoescutelo con puntuaciones variables $(i=0-2 d)$ menos densa en el disco; puntuaciones variables, de menor tamaño en escutelo y metanoto; propodeo ligeramente puntuado-reticulado; área hipoepimeral y mesepisterno con puntuaciones variables (i=1-2.5d). Metasoma: peciolo con puntuaciones variables, escasas, aisladas; T1 impuntuado, pulido; resto de los tergos con escasas puntuaciones, pequeñas. Esternos mayormente impuntuados, con escasas puntuaciones variables, excepto T6 con puntuaciones irregulares, groseras.

Pubescencia. Blanca, excepto en clípeo, algunos pelos aislados en cabeza y esternos, negros; frente con pelos blanco, negros y otros bicolores, con la base negra hasta la mitad continuándose con blanco. Pubescencia blanco-gris apretada en clípeo y área paraocular. Pelos largos, rectos, ligeramente aislados (3-4 DOM) en cabeza y mesosoma; más largos en gena, propodeo y áreas ventrales y laterales del mesosoma. Pubescencia escasa en metasoma, excepto en peciolo, con algunos pelos aislados en esternos, más abundantes al final del metasoma (en T6 y S6).

Estructura. Mandíbula con dos dientes distales, el diente interno no llega a dividirse por surco bien definido (fig. 23) (en hembras viejas las mandíbulas aparecen gastadas, el diente más distal no es puntiagudo). Clípeo engrosado en el borde libre, con una pequeña muesca en el centro (fig. 23). Peciolo casi tan largo como el metafémur.

\section{MACHO: DESCONOCIDO}

Tipos. Holotipo hembra: HISPANIOLA, Dominican Republic, Prov. La Altagracia, Cueva de Berna, Boca de Yuma, 6.vi.1986, cols. R. Miller \& L. Stange (FSCA). Paratipos hembras: Puerto Rico, Aibonito, 27.v.1915, sin nombre del recolector, Chlorion (Isodontia) aztecum (Sauss.) var. cinereum (Fernand.) det. Dow, Isodontia poeyi ? Pate det. A. S. Menke (NMNH); Aguadilla, playa, 22.ix.1979, col. J. Santiago (JAG). Hispaniola, República Dominicana, Hato Mayor, San Rafael, 27.iv.2001, col. H. Matzusawa (MNHNSD); Boca de Yuma, Parque Nacional de Este, 29.i.2001, cols. J. A. Genaro, E. Gutiérrez \& G. Alayón (JAG).

Etimología. Referente al área que ocupa, las Antillas.

Distribución. Hispaniola y Puerto Rico.

Comentario. Este constituye el primer registro del género para la Hispaniola y Puerto Rico. Isodontia p. poeyi Pate, 1948 vive en Cuba e Isla de la Juventud. A continuación se aclaran algunos aspectos sobre la deposición de los tipos primarios de la subespecie nominal. El holotipo macho está depositado en la Academia de Ciencias Naturales de Filadelfia; un paratipo también macho, depositado en la colección de la Estación Experimental Agrícola de Santiago de Las Vegas, La Habana, no aparece en la colección entomológica del Instituto de Ecología y Sistemática, donde fueron llevadas las colecciones de aquella institución. La hembra de la subespecie nominal no está descrita.

El estudio de los machos y/o el ADN de I. poeyi antillana ssp. nov. pudiera arrojar mayores diferencias que impliquen la elevación al rango de especie. 


\author{
Familia Crabronidae \\ Subfamilia Pemphredoninae \\ Pseneo alayoni sp. nov.
}

(figs. 24-26)

Diagnosis. Tegumento negro con pubescencia blanca excepto amarillo claro en placa pigidial. Una combinación de caracteres como la forma del clípeo y área pigidial, y la esculturación del propodeo, la separan de otras especies neotropicales.

Diagnosis. Black with white pubescence except light yellow hairs in pigidial plate. A combination of characters such as form of the clypeus and pigidial plate, and surface sculpturing of propodeum separates the new species from the rest of the Neotropical ones.

\title{
DESCRIPCIÓN
}

\section{HEMBRA}

Longitud corporal total aproximada: 18.2-21.2 mm. Longitud ala anterior: 16.0-17.5 mm.

Coloración. Tegumento negro con algo de castaño rojizo en mandíbulas y tarsos. Ala ligeramente ahumada con celdilla marginal oscurecida; nervaduras, estigma y celdilla costal castaño oscuro.

Esculturación. Cabeza: clípeo, área supraclipeal, frente y vértex con puntuaciones irregulares $(\mathrm{i}=0-1 \mathrm{~d})$, grandes y pequeñas; gena con puntuaciones irregulares, pequeñas $(\mathrm{i}=1.5-4 \mathrm{~d})$. Mesosoma: mesoescutelo con puntuaciones irregulares $(\mathrm{i}=0-5 \mathrm{~d})$, mayores que en la gena, menores en el disco, dos carenas paralelas longitudinales que tocan el margen anterior y se extienden más allá del centro; alrededor de estas carenas la puntuación es menor y aparecen ligeras arrugas longitudinales a ambos lados; escutelo con puntuaciones irregulares, más unidas en margen anterior y posterior $(\mathrm{i}=0-4 \mathrm{~d})$, más pequeñas sobre el margen posterior; metanoto con puntuaciones groseras, unidas; tégula imbricada con puntuaciones irregulares en el margen anterior, impuntuada posteriormente; mesepisterno con puntuaciones unidas, alargadas y otras más pequeñas, aisladas hacia el área posterior; área basal del propodeo con estrías subparalelas que tocan el margen posterior; superficie posterior del propodeo con estrías subparalelas longitudinales que se dirigen hacia afuera, creando celdillas en área intermedia, estrías subparalelas transversales hacia el margen posterior (fig. 25). Metasoma: peciolo pulido, con línea dorsal longitudinal de micro-puntuaciones; con dos surcos, desarrollados, en área ventral. Tergos pulidos, imbricados, con micro-puntuaciones irregulares, aisladas, mayores en T4-T6; puntuaciones groseras, mayores en T6, de donde emerge un pelo de cada una. Área pigidial imbricada con puntuaciones irregulares ( $\mathrm{i}=0-4 \mathrm{~d})$, más densas hacia el ápice (fig. 26). Esternos imbricados con puntuaciones aisladas, irregulares y otras algo mayores distribuidas irregularmente; T6 con puntuaciones mayores, más profundas.

Pubescencia. Pubescencia blanca excepto amarillo claro en placa pigidial. Blanco brillante apretada (=DOM) en área supraclipeal y paraocular inferior; menos densa en base de las mandíbulas, del clípeo y frente. Pelos cortos $(=\mathrm{DOM})$ cubriendo uniformemente vértex y gena. Pelos menos abundantes en áreas dorsales del mesosoma, excepto metanoto y área basal del propodeo, donde es más gruesa. Hilera de pelos finos $(<\mathrm{DOM})$, separados, a ambos lados del peciolo. T1-T4 con parches de pubescencia apretada, recostada a los lados; pubescencia amarillo claro recostada (>DOM) en área pigidial, convergiendo en hileras hacia el ápice; erecta, aislada a los lados de la placa pigidial. 
Estructura. Mandíbula bidentada. Clípeo abultado con margen engrosado, estrechándose en la zona media, borde libre recto (fig. 24). Peciolo ligeramente más largo que metafémur. Área pigidial estrecha, triangular (fig. 26).

\section{MACHO: DESCONOCIDO}

Tipos. Holotipo hembra. HISPANIOLA, República Dominicana, Parque Nacional A. Bermúdez, Antón Sape Bueno, Trillo de la Hispaniola, xii. 2008, col. J. A. Genaro (FSCA).

Etimología. Dedicado al amigo y especialista en arañas Giraldo Alayón (MNHNCu), por sus valiosas contribuciones en el campo de la sistemática de invertebrados y la divulgación de la historia natural antillana.

Distribución. La Hispaniola, Antillas Mayores.

Comentario. Ejemplar recolectado frente a una pared vertical de sustrato, posiblemente relacionado con la nidificación. Este constituye el primer registro del género para la isla de La Hispaniola. Genaro y Alayo (2001) registraron por primera vez al género para Cuba y describieron dos especies nuevas, basados en escasos ejemplares, ya que estas especies son raramente observadas y recolectadas, habitando en áreas bien conservadas, ecológicamente.

\section{AGRADECIMIENTOS}

A David Carmean (Simon Fraser University, Canadá) por facilitar y compartir la información sobre la especie de Taeniogonalos de la Hispaniola. A Esteban Gutiérrez por la amable ayuda brindada en la consulta taxonómica de los ejemplares de T. gundlachii y Cerceris spp. depositados en el MNHNCu. Sophie Cardinal y J. L. Fernández (CNC) permitieron y facilitaron, para su estudio, el préstamo del sexo opuesto de Taeniogonalos. Víctor González (San Juan, Puerto Rico) financió las expediciones a República Dominicana. Agradezco también a Ivonne Arias (Grupo Jaragua) por el apoyo brindado durante el trabajo de campo en República Dominicana. Carlos Suriel y Celeste Mir facilitaron el acceso a las colecciones del MNHNSD y su documentación, para el estudio de ejemplares. Gino Nearns (Purdue University, EE. UU.) amablemente envió literatura. A mi esposa C. Juarrero por la preparación y diseño de las láminas. Luis F. de Armas gustosamente revisó la primera versión del manuscrito.

\section{LITERATURA CITADA}

Alayo, P. 1968. Estudios sobre los himenópteros de Cuba. I. Subfamilia Philanthinae (Familia Sphecidae). Poeyana, 54: 1-23.

Alayo, P. 1974. Introducción al estudio de los himenópteros de Cuba, superfamilia Bethyloidea. Serie Biológica, Academia de Ciencias de Cuba, 52: 1-24.

Carmean D. y L. S. Kimsey. 1998. Phylogenetic revision of the parasitoid wasp family Trigonalidae (Hymenoptera). Systematic Entomology, 23: 35-76.

Elliott, N. B., W. M. Elliott y P. Salbert. 1981. Nesting behavior of Cerceris zonata (Hymenoptera: Philanthidae). Annals of the Entomological Society of America, 74: 127-129. 
Genaro, J. A. 2009. Dos especies nuevas de Cerceris de las Antillas Mayores Hymenoptera: Apoidea, Crabronidae), con notas sobre su historia natural. Solenodon, 8: 99-117.

Genaro, J. A. y C. S. Sánchez. 1993. Conducta de nidificación de Cerceris cerverae, C. cubensis y C. festiva en Cuba (Hymenoptera: Sphecidae). Caribbean Journal of Science, 29: 39-43.

Genaro, J. A. y P. Alayo. 2001. Dos especies nuevas de Pseneo para Cuba (Hymenoptera: Sphecidae, Pemphredroninae). Cocuyo, 10: 2-3.

Harris, R. A. 1979. A glossary of surface sculpturing. Occasional Papers in Entomology, Department of Food and Agriculture, Division of Plant Industry California, 28: 1-31.

Nelson, G. H. y C. L. Bellamy. 2004. A revision of the genus Paratyndaris Fisher, 1919 (Coleoptera: Buprestidae: Polycestinae). Zootaxa, 683: 1-80.

Salbert, P. y N. B. Elliott. 1979. Observations on the nesting behavior of Cerceris warlingensis (Hymenoptera: Sphecidae, Philanthinae). Annals of the Entomological Society of America, 72: 951-55.

Scullen, H. A. 1965. Review of the genus Cerceris in America North of Mexico (Hymenoptera: Sphecidae). Proceedings of the United States National Museum, 116: 333-547.

Scullen, H. A. 1972. Review of the genus Cerceris Latreille in Mexico and Central America (Hymenoptera: Sphecidae). Smithsonian Contributions to Zoology, 110: 1-121.

Smith, D. R., D. H. Janzen, W. Hallwachs y M. A. Smith. 2012. Hyperparasitoid wasps (Hymenoptera, Trigonalidae) reared from dry forest and rain forest caterpillars of Area de Conservación Guanacaste, Costa Rica. Journal of Hymenoptera Research, 29: 119-144.

Snelling, R. R. 2005. Wasps, ants, and bees: Aculeate Hymenoptera. In J. Lazell (Ed.) Island: Fact and Theory in Nature. Pp. 283-296. University of California Press, Berkeley, USA.

Yong, S. y D. Breto. 2015. Nuevos reportes de bupréstidos (Coleoptera: Buprestidae) como presas de la avispa Cerceris cerverae (Hymenoptera: Crabronidae). Boletín de la Sociedad Entomológica Aragonesa, 56: 370-372. 